\title{
Arterial Injury Associated with Tension-Free Vaginal Tapes- SECUR Procedure Successfully Treated by Radiological Embolization
}

\author{
Yun Seok Jung, Joon Ho Lee, Tae Seung Shin, Chang Hee Han, Sung Hak Kang, Yong Seok Lee \\ Department of Urology, The Catholic University of Korea School of Medicine, Seoul, Korea
}

\begin{abstract}
Various postoperative complications have been reported after the use of tension-free vaginal tapes (TVT). The transobturator approach was introduced to minimize the potential complications. The next generation of recently introduced TVT-SECUR is intended to minimize the incidence of complications. Herein we report a case of internal pudendal artery injury sustained during this procedure that was successfully treated by radiological embolization. Angiography with vessel embolization, when available, should be considered when the arterial injury is suspected.
\end{abstract}

Keywords: TVT-SECUR; Urinary incontinence; Embolization; Complication

The tension-free vaginal tape (TVT) procedure is the most commonly used method for the treatment of stress urinary incontinence. Although this method is safe and simple, potentially serious complications in the bladder, intestines, and larger vessels associated with the retropubic passage of the needles can still occur. For the alternative transobturator technique, the possible complications include pain in the leg or groin, bladder perforation, and hematoma [1].

Therefore, less invasive methods have been developed, one of which is the Gynecare TVT-SECUR System (Ethicon Inc., Somerville, NJ, USA) [2]. The aim of this procedure is to anchor the tape in the obturator muscle to avoid a full needle passage, thus possibly reducing the associated complications as described above.

We report here a case of a serious bleeding complication following the TVT-SECUR procedure caused by an injury to the internal pudendal artery.

\section{CASE REPORT}

A 60-year-old woman presented with bothersome stress urinary incontinence. Evaluation of her current complaint revealed a mobile urethra, stable cystometrogram, and complete bladder emptying. She had a positive cough stress test with a Valsalva leak point pressure of $119 \mathrm{~cm} \mathrm{H}_{2} \mathrm{O}$ with $150 \mathrm{~mL}$ of fluid in the bladder.

She underwent the TVT-SECUR procedure under general anesthesia. There were no complications intraoperatively. One hour after surgery, she complained of lower abdominal pain, a feeling of lower abdominal distension, and nausea. In addition, her systolic blood pressure decreased gradually to $70 \mathrm{mmHg}$. Her hemoglobin changed from $12.7 \mathrm{~g} / \mathrm{dL}$ preoperatively to 8.3 g/dL about 2 hours postoperatively.

Computed tomography (CT) revealed a retropubic hyperdense hematoma sized up to $10 \mathrm{~cm} \times 9 \mathrm{~cm} \times 8 \mathrm{~cm}$ and active contrast leakage from the left internal pudendal artery (Fig. 1).
Corresponding author: Yong Seok Lee

Department of Urology, Uijeongbu St. Mary's Hospital, The Catholic University of Korea School of Medicine, 65-1 Geumo-dong, Uijeongbu 480-717, Korea Tel: +82-31-820-3588 / Fax: +82-31-847-6133 / E-mail: ysleemd@catholic.ac.kr Submitted: November 22, 2010 / Accepted after revision: December 9, 2010
This is an Open Access article distributed under the terms of the Creative Commons Attribution Non-Commercial License (http://creativecommons.org/licenses/by-nc/3.0/) which permits unrestricted non-commercial use, distribution, and reproduction in any medium, provided the original work is properly cited. 


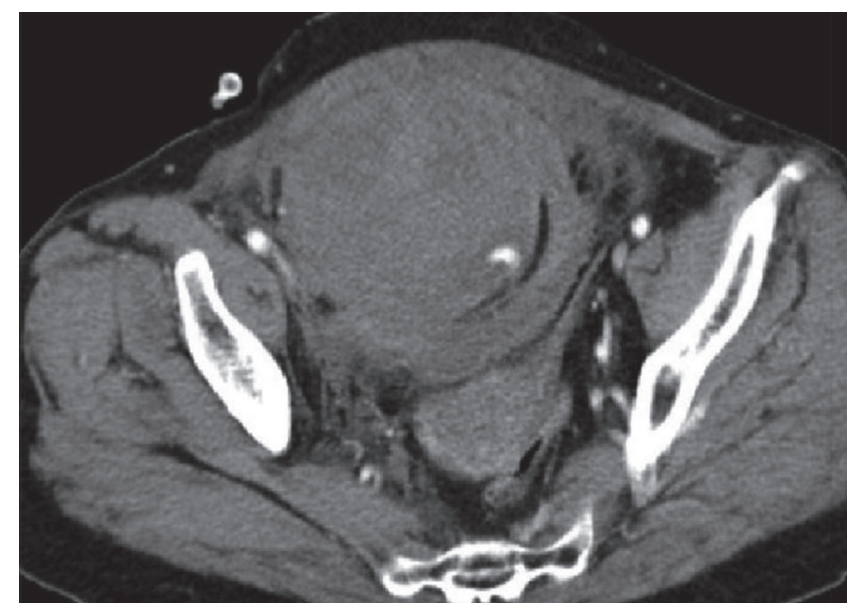

Fig. 1. Postoperative pelvic computed tomography reveals large pelvic hematoma and active contrast leakage from left internal pudendal artery.

Successively, arteriography was performed. The arteriography also confirmed left internal pudendal arterial injury, which was successfully treated by selective embolization with a gelatin sponge (Gelfoam, Pfizer Inc., New York, NY, USA)) with 25\% $n$-butyl-cyanoacrylate (Fig. 2). There were no postprocedure complications.

The patient's postoperative course was uneventful requiring neither blood transfusion nor surgical removal of the hematoma. Two years after surgery, her stress incontinence was resolved, and she complained of mild urge incontinence.

\section{DISCUSSION}

Since the TVT procedure was introduced, it has become the gold standard for the treatment of female stress urinary incontinence. Although this method is minimally invasive, various complications have been reported even though the complication rate is low. New minitapes, such as TVT-SECUR, may be a way to reduce such perioperative complications. The TVT-SECUR system was developed with the intention of further reducing the invasiveness of the surgical procedures by avoiding the passage of the needle carriers through the retropubic or obturator regions [2]. The advantages of this new technique are thus related to the short passage of the needles, which minimizes the risk of vascular, nerve, or visceral injury. The TVT-SECUR system was designed to overcome two of the perioperative complications reported with the use of the TVT-obturator: thigh pain and bladder outlet obstruction $[3,4]$. These complications were addressed by tailoring the tape to a length of only $8 \mathrm{~cm}$ and anchoring the

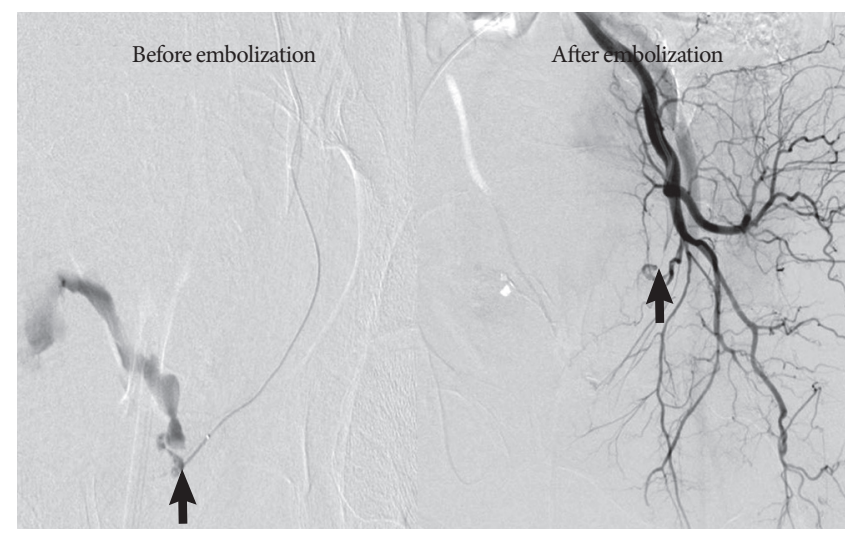

Fig. 2. Pelvic arteriography before and after embolization. Arrows indicate left internal pudendal artery.

tape edges into the internal obturator muscle, rather than passing it through the obturator foramen, muscle, and membrane. However, a possible reason for hemorrhagic complications of the TVT-SECUR procedure may be the scalpel-shaped tip of the inserter, which can cut the vessels.

Minor intraoperative bleeding from the initial vaginal dissection can usually be controlled with direct pressure on the paraurethral and retropubic areas, held for 5 to 10 minutes and followed by vaginal packing. Greatly increased bleeding that results in a retropubic hematoma usually arises from a venous injury during trocar passage, and up to $2.8 \%$ of these cases may require transfusion [5].

Depending on the size of the hematoma, signs and symptoms may include pain, urgency and frequency from bladder compression, a suprapubic mass, hypotension, tachycardia, lightheadedness, vaginal and abdominal tenderness, and a decrease in hematocrit.

Interestingly, specified bleeding from an internal pudendal artery has not been reported previously with the TVT or TVTobturator technique. Khandwala et al. [6] reported in research carried out on 141 cases of TVT-SECUR that no injuries to the vessels and no hematoma were observed. Meschia et al. [7] demonstrated that no bleeding or hematoma occurred during their 91 cases of the TVT-SECUR procedure. However, severe bleeding from the internal obturator muscle and injury of the corona mortis after TVT-SECUR have been reported [8,9].

If abnormal abdominal pain or abdominal distension appears after the TVT-SECUR procedure, a CT scan should be performed. A hematoma in the pelvic cavity is not palpable during a vaginal examination. The patient should also be carefully examined if other complications occur, such as buttock or groin 
pain, signs of shock, vaginal bleeding, or urinary retention.

To decrease the risk of vascular injury, the pelvic surgeon should pay attention to proper technique during the approach and have a high clinical suspicion of complications under the appropriate circumstances.

\section{CONFLICT OF INTEREST}

No potential conflict of interest relevant to this article was reported.

\section{REFERENCES}

1. Nilsson CG. Latest advances in TVT tension-free support for urinary incontinence. Surg Technol Int 2004;12:171-6.

2. Molden SM, Lucente VR. New minimally invasive slings: TVT Secur. Curr Urol Rep 2008;9:358-61.

3. Neuman M. TVT and TVT-Obturator: comparison of two operative procedures. Eur J Obstet Gynecol Reprod Biol 2007;131:89-92.

4. Neuman M. TVT-obturator: short-term data on an operative pro- cedure for the cure of female stress urinary incontinence performed on 300 patients. Eur Urol 2007;51:1083-7.

5. Kobashi KC, Govier FE. Perioperative complications: the first 140 polypropylene pubovaginal slings. J Urol 2003;170:1918-21.

6. Khandwala S, Jayachandran C, Sengstock D. Experience with TVTSECUR sling for stress urinary incontinence: a 141-case analysis. Int Urogynecol J Pelvic Floor Dysfunct 2010;21:767-72.

7. Meschia M, Barbacini P, Ambrogi V, Pifarotti P, Ricci L, Spreafico L. TVT-secur: a minimally invasive procedure for the treatment of primary stress urinary incontinence. One year data from a multicentre prospective trial. Int Urogynecol J Pelvic Floor Dysfunct 2009;20:313-7.

8. Masata J, Martan A, Svabík K. Severe bleeding from internal obturator muscle following tension-free vaginal tape Secur hammock approach procedure. Int Urogynecol J Pelvic Floor Dysfunct 2008; 19:1581-3.

9. Larsson PG, Teleman P, Persson J. A serious bleeding complication with injury of the corona mortis with the TVT-Secur procedure. Int Urogynecol J Pelvic Floor Dysfunct 2010;21:1175-7. 